\title{
Neuroimages and Neuropathology of a Stroke-Like Cerebral Lymphomatoid Granulomatosis
}

\author{
Massimo Marano (D), Chiara Brunelli, Costanza De Marco, Maurizio Martini, \\ Carlo Cosimo Quattrocchi, Libero Lauriola, Vincenzo Di Lazzaro
}

Keywords: Brain neoplasm, Lymphoma, Vasculitis, Brain stereotactic biopsy, Glioma, stroke mimic

doi: $10.1017 / \operatorname{cjn} .2020 .133$

Can J Neurol Sci. 2021; 48: 114-115

A 70-year-old man presented to the Emergency Department reporting the acute onset of non-fluent aphasia, hyposthenia, and hemi-anesthesia of the right body. Brain computerized tomography revealed a subcortical hypodense lesion in the middle cerebral artery territory. Neck ultrasounds of internal and external carotid arteries and of the vertebral arteries showed a focal moderate stenosis of the left internal carotid artery due to a soft atheromasic plaque. These findings that were initially consistent with a diagnosis of an ischemic stroke were not confirmed by magnetic resonance (MR). The latter showed an hyperintense lesion on FLAIR and T2-weighted sequences located in the left centrum semiovale, corona radiata, and thalamus, with a well-defined regular rim and a mild compressive effect on the lateral ventricle, with diffusivity restriction but without $\mathrm{ADC}$ reduction and with a punctate and serpiginous gadolinium enhancement on T1 sequences (Figure 1). Within the first day of observation, the patient started complaining progressive mental deterioration, in absence of any other possible causes, and a total body CT scan excluded any other organ involvement. Patient was then referred to the neurosurgeon in order to perform a brain biopsy. The neuropathology was compatible with the diagnosis of cerebral lymphomatoid granulomatosis (LG) (Figure 1).

Central nervous system (CNS) lymphoproliferative disorders include the primary diffuse large B-cell lymphoma of the CNS and a variety of other lymphomas and related lymphoproliferative conditions, such as the LG. LG is a rare angiocentric and angiodestructive lymphoreticular proliferative disorder. ${ }^{1}$ It is an EBV-driven systemic disease, which usually involves lungs followed by the skin and the brain. Isolated CNS LG (CLG) is an extremely rare condition, and it is only anecdotally reported in literature and poorly characterized on a neuroradiological and clinical point of view. Its pathogenesis and biological behavior are debated and, according to some authors, the isolated CLG is not related to EBV and shows a better prognosis overall. ${ }^{1,2}$ Our case is in line with such observation since the immunophenotype - in situ hybridization analysis of EBV-encoded small RNAs (EBER ISH) - of the pathological specimen was negative for the EBV virus, whilst the immunoglobulin $\mathrm{IgH}$ gene (FR3/JH) and the $\mathrm{T}$-cell receptor $(\mathrm{TcR} \gamma)$ rearrangement showed a polyclonal pattern as documented in LG cases with negative EBV. ${ }^{1}$ On the

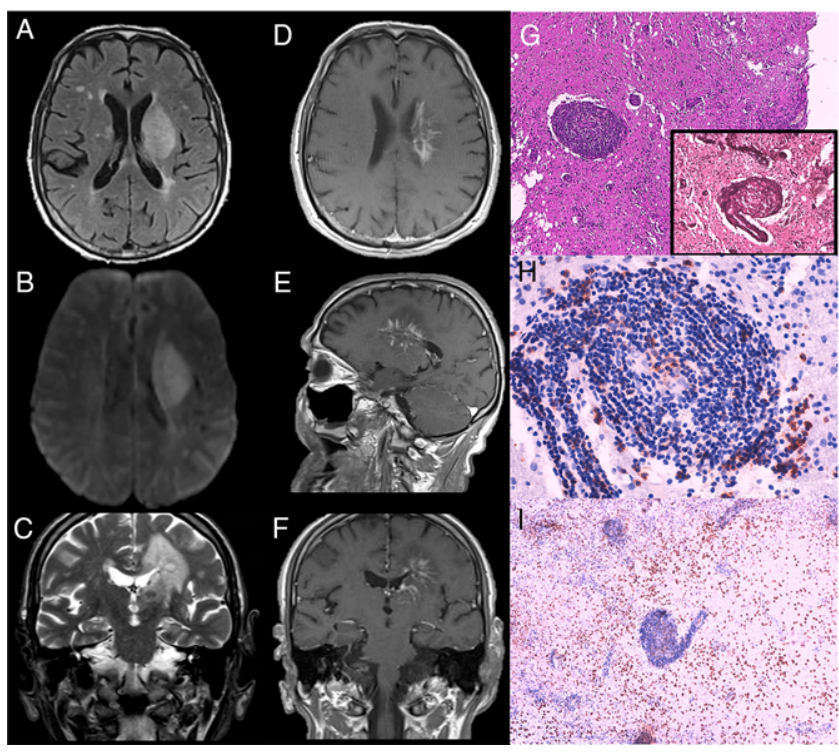

Figure 1: An undefined edematous lesion in the left thalamus/corona radiata seen at the brain MRI axial FLAIR (A), axial DWI (B), and coronal T2-weighted sequences as a hyperintense lesion $(C)$ with a serpiginous gadolinium enhancement featuring its core at axial $(D)$, sagittal (E), and coronal (E) T1-weighted images. The hematoxylineosin stain showed a cerebral tissue with vascular structures surroundend by a polymorphous lymphoid infiltrate $(G, 100 \times)$. In detail at higher magnification (inset in $G, 200 \times$ ) the reticulin stain demonstrated the presence of dispersed reticular fibers due to the angiodestructive behavior of the disease. Immunostaining showed few and isolated CD2O activated $B$ cells $(H, 400 \times)$ in an abundant CD3 lymphocyte background $(I, 100 \times)$.

From the Unit of Neurology, Neurophysiology, Neurobiology, Department of Medicine, Università Campus Bio-Medico di Roma, via Álvaro del Portillo 21, 00128 Rome, Italy (MM, VDL); Department of Anatomic Pathology and Histology, "A. Gemelli" University Polyclinic Foundation, Rome, Italy (CB, CDM, MMartini, LL); and Radiology Unit, Università Campus Bio-Medico di Roma, via Álvaro del Portillo 21, 00128 Rome, Italy (CCQ)

Received January 13, 2020. Final Revisions Submitted May 18, 2020. Date of ACCEPTANCE June 25, 2020.

Correspondence to: Massimo Marano, Unit of Neurology, Neurophysiology, Neurobiology, Department of Medicine, Università Campus Bio-Medico di Roma, via Álvaro del Portillo 21,00128 Rome, Italy. Email: m.marano@unicampus.it 
other side, the WHO LG grading relies on the amount of EBV positive cells and the presence of a secondary CNS involvement predicts a lower life expectancy.

Differentiating primary lymphoproliferative disorders from other brain lesions could be difficult, and the histological analysis results essential in the diagnosis. The presence of an angiodestructive process makes it possible the differentiation with brain lymphomas which share with the CLG the angioinvasive features. ${ }^{3}$ Pathognomonic neuroimages have not been ever identified, but the most common MR finding is a linear or a "multiple punctuate" enhancement along perivascular spaces. Large mass-like lesions, ring-enhancement lesions, and leptomeningeal involvement have also been reported. ${ }^{4,5}$ CLG lacks any clinical or radiological specific sign. Vasculitis, demyelinating, infectious, and neoplastic disorders could present similar features making only histopathology diagnostic, so far. The clinical presentation is highly variable, and the available cases are described as subacute disorders leading to a progressive neurological deterioration. Patients generally present with headache, seizures, altered consciousness, motor progressive deterioration and dementia, and cranial nerve palsies have also been described. A stroke-like presentation is very rare. ${ }^{1,2}$ This could be justified by the vascular and perivascular destructive process, which produced a microvascular damage with consecutive ischemic changes of the surrounding tissue, thus explaining the possibility of an acute stroke-like clinical presentation.

\section{Disclosures}

The authors have no conflicts of interest to declare.

\section{Ethical Approval}

The research has been given ethical approval and is conducted in accordance with the declaration of Helsinki.

\section{Statement of Authorship}

MM, CCQ, and VDL collected clinical and radiological data; CB, CDM, MMartini, and LL provided brain pathology samples and description.

\section{REFERENCES}

1. Lucantoni C, De Bonis P, Doglietto F, et al. Primary cerebral lymphomatoid granulomatosis: report of four cases and literature review. J Neurooncol. 2009;94:235-42.

2. Roschewski M, Wilson WH. Lymphomatoid granulomatosis. Cancer J. 2012;18:469-74.

3. Sugita Y, Muta H, Ohshima K, et al. Primary central nervous system lymphomas and related diseases: Pathological characteristics and discussion of the differential diagnosis. Neuropathology 2016;36:313-24.

4. Patsalides AD, Atac G, Hedge U, et al. Lymphomatoid granulomatosis: abnormalities of the brain at MR imaging. Radiology 2005;237:265-73.

5. Tateishi U, Terae S, Ogata A, et al. MR imaging of the brain in lymphomatoid granulomatosis. AJNR Am J Neuroradiol. 2001;22:1283-90. 